\title{
Proposal: Let's Develop a Community Consensus K-ratio Database
}

Nicholas Ritchie $^{1}$, Dale Newbury ${ }^{1}$, Emma Bullock ${ }^{2}$, Paul Carpenter ${ }^{3}$, John Donovan ${ }^{4}$, John Fournelle ${ }^{5}$, Anette von der Handt ${ }^{6}$, Heather Lowers ${ }^{7}$, Aurélien Moy ${ }^{8}$, Owen Neill ${ }^{9}$ and Edward Vicenzi ${ }^{10}$

${ }^{1}$ National Institute of Standards and Technology, Gaithersburg, Maryland, United States, ${ }^{2}$ Carnegie Institution for Science, Washington, District of Columbia, United States, Washington University, St. Louis, Missouri, United States, ${ }^{4}$ University of Oregon, Eugene, Oregon, United States, ${ }^{5}$ University of Wisconsin, Madison, Madison, Wisconsin, United States, ${ }^{6}$ University of Minnesota, Minneapolis, Minnesota, United States, ${ }^{7}$ U.S. Geological Survey, DENVER, Colorado, United States, ${ }^{8}$ Department of Geoscience, University of Wisconsin-Madison, Madison, Wisconsin, United States, ${ }^{9}$ University of Michigan, Ann Arbor, Michigan, United States, ${ }^{10}$ Smithsonian Institution, Suitland, Maryland, United States

Electron probe microanalysis (EPMA), whether by wavelength-dispersive spectrometer or energy dispersive spectrometer, is a method for measuring the composition of a material. However, EPMA is an indirect technique. The fundamental measurand in EPMA is the X-ray intensity. Materials are compared through the ratio of X-ray intensities measured under similar conditions on the unknown and a standard called the k-ratio. The k-ratio is converted to a measure of composition through an implicit multivariate measurement model [1], called matrix correction, in which we compute the anticipated k-ratio for a specified element and X-ray line in a specified material and compare it with the measured k-ratio. The estimated composition is adjusted through a non-linear optimization process, called iteration, until the computed k-ratio equals the measured k-ratio.

Point 1: The community is increasingly observing pathological measurements in which this inference is breaking down. We are discovering there are elements in certain materials for which we are not able to accurately compute the measured k-ratio. While problems with measurements using the L3-M5 transition in transition metals [2] or the M-lines in rare-earth metals [3] have been long appreciated, there has been a renewed interest recently in systems with iron [4] and nickel [5]. Similarly, the wavelength community has long used area peak factors to accommodate certain light elements for which the shape and position of the characteristic X-ray line varies from material to material [6]. While it might eventually be possible to address these materials with more sophisticated matrix correction models which account for complex solid-state effects, it may be easier and more accurate, even then, to keep a shared database of k-ratios for these materials.

Point 2: Even for non- pathological measurements, the accuracy of EPMA compositional measurements is usually limited by the accuracy of the matrix correction model. To accommodate this, many careful microanalysts often use compositional standards similar in composition to their unknown. This process minimizes the magnitude of the matrix corrections and typically leads to more reliable measurements. However, it also requires that the laboratory have ready access to a material of known (certified) composition similar to the unknown. In labs which analyze a variety of materials, this can be impractical.

There is a way that the community can address both of these challenges, and others. Under a basic set of assumptions (including a common take-off angle and beam energy), the k-ratio is a universal 
property. Detector efficiency, solid angle, window type, detector vendor and many other hard-to-control parameters should not matter. The k-ratio measured on one instrument should equal, to within measurement precision, the k-ratio measured on another instrument.

The proposal is that we should, as a community, start collecting and curating a shared database of kratios. The utility of this database would arise because the k-ratio of material A with respect to material $\mathrm{B}$ can be determined through an intermediary material $\mathrm{C}$. If $\mathrm{k}_{\mathrm{AC}}=\mathrm{I}_{\mathrm{A}} / \mathrm{I}_{\mathrm{C}}$ and $\mathrm{kBC}=\mathrm{I}_{\mathrm{B}} / \mathrm{I}_{\mathrm{C}}$, then $\mathrm{k}_{\mathrm{AB}}=\mathrm{I}_{\mathrm{A}} / \mathrm{I}_{\mathrm{B}}$ $=\left(\mathrm{I}_{\mathrm{A}} / \mathrm{I}_{\mathrm{C}}\right) /\left(\mathrm{I}_{\mathrm{B}} / \mathrm{I}_{\mathrm{C}}\right)=\mathrm{k}_{\mathrm{AC}} / \mathrm{kBC}_{\mathrm{BC}}$. If there is agreement about $\mathrm{C}$, then one lab can measure $\mathrm{k}_{\mathrm{AC}}$ and another can measure $\mathrm{k}_{\mathrm{BC}}$ and the first lab can determine $\mathrm{k}_{\mathrm{AB}}$ without ever having material $\mathrm{B}$ in their lab or ever measuring it directly. It becomes possible to share k-ratios between labs and unnecessary to share the material $B$.

The focus must be on community consensus.

- Community - No one community member should be forced to / entrusted to populate the database. The choice of k-ratios and the measurements should reflect the interests of the community. Community members should be able to contribute easily. The database should be free-and-open to all whether academic, governmental, commercial or instrument vendor. The implementation should be open-source under a liberal license and the data should be readily available both in bulk and through a query-able web application programming interface (API).

- Consensus - The correctness of individual k-ratios should not be determined by fiat but rather by a process in which as a result of many independent measurements, the community comes to a consensus value. The degree to which the community can agree on common "exchange references" (the material $\mathrm{C}$ above), the more useful the database becomes. The community should consider carefully the choice of exchange references for each element and transition.

Initially, the project involves building, as a community, the infrastructure necessary to administer the database and building the support necessary to populate it once it becomes available. The community must be careful to design the database to contain enough detail to be useful and yet not so much data as to be unwieldy to manage or to populate. We need to address both the wavelength- and energy-dispersive detector technologies. We will need to develop a data curation backend, a web programmer's API and a web-based user interface for both uploading and accessing data. The implementation will need to be hosted somewhere in a free-and-open manner. Whoever hosts it, the data and the infrastructure must be free, open and transparent. We will welcome instrument vendors to use the web API to integrate the database with their products. We will welcome research groups and vendors to use the data to design better quantification algorithms and further microanalytical metrology.

However, the success of this project depends on contributions from individuals within the electron excited microanalysis community. We expect that this database has the potential to revolutionize microanalysis. We hope that you will join with us to build and populate this community enhancing enterprise.

\section{References}

[1] BIPM, IEC, ILAC IFCC, IUPAC ISO, and OIML IUPAP. (2011) "Evaluation of measurement dataSupplement 2 to the 'Guide to the expression of uncertainty in measurement' - Extension to any number of output quantities." JCGM 102 (2011). 
[2] Nagel, D. (1968). Absorption edge effects in electron probe microanalysis. In Quantitative Electron Probe Microanalysis, Heinrich, K.F.J (Ed.), vol. 298, pp. 189-196. Washington, DC: National Bureau of Standards Special Publication.

[3] Fischer, D. W. and Baun, W. L. (1967) "Self-Absorption Effects in the Soft X-Ray M $\alpha$ and M $\beta$ Emission Spectra of the Rare Earth Elements" J. Appl Physics. 38 4830-4836.

[4] Gopon, P., Fournelle., J., Sobol, P. \& Llovet, X. (2013). Low-voltage electron-probe microanalysis of Fe-Si compounds using soft X-rays. Microsc Microanal 19, 1698-1708.

[5] Llovet, X. Pinard, P.T., Heikinheimo, E. and Richter, S. (2016) Electron Probe Microanalysis of Ni Silicides Using Ni-L X-Ray Lines, Microsc. Microanal. 22, 1233-1243

[6] Bastin G.F., Heijligers H.J.M. (1991) Quantitative Electron Probe Microanalysis of Ultra-Light Elements (Boron-Oxygen). In: Heinrich K.F.J., Newbury D.E. (eds) Electron Probe Quantitation. Springer, Boston, MA 